\title{
Prediction of Ship Traffic Volume in Jiujiang Port Based on Genetic Wavelet Neural Network
}

\author{
Shouwei XIE ${ }^{1, a}$ and Yadong YANG ${ }^{2}$ \\ ${ }^{1}$ College of Wuhan University of Technology, Hubei Provincial Key Laboratory of Inland navigation technique, china \\ ${ }^{2}$ College of Wuhan University of Technology, Hubei Provincial Key Laboratory of Inland navigation technique, china
}

\begin{abstract}
In recent years, the traffic volume of the Yangtze River has increased dramatically. In order to provide more favorable assistance to port planning and traffic management, the accuracy of port ship traffic volume prediction is very important. In this paper, genetic algorithm and wavelet analysis and neural network are used to construct the genetic wavelet neural network model prediction model, and BP neural network prediction model is established. The ship volume of Jiujiang Port is used as experimental data to simulate and analyze. The results show that the prediction accuracy of the genetic wavelet neural network prediction model is significantly higher than that of the BP neural network prediction model. It is proved that the genetic wavelet neural network has broad application prospects for ship traffic flow prediction in the Yangtze River port. This method has practical application significance.
\end{abstract}

\section{The introduction}

These In recent years, the domestic economic development trend has become better and better, and various industries have also developed rapidly. Among them, the development of waterways has become more and more prominent. In order to meet the needs of economic growth, the traffic volume of the Yangtze River has increased sharply in recent years, and the development of China's waterway transportation is prominent at this stage. Contradiction is the contradiction between advanced navigation technology and backward port transportation management. In order to solve this contradiction, it is necessary to strengthen port ship management and preparation[1]. Accurately predicting the traffic volume of port ships can alleviate the pressure of shipping traffic and the maritime department and port transportation management departments. It can also formulate more detailed berthing, berthing and piloting plans to fundamentally solve the problem of congestion in port transportation and improve the application of ships. effectiveness.

There are two general aspects to the prediction of ship traffic volume:

(1) Prediction of time series;

(2) Predictions in terms of impact factors;

The specific methods include regression prediction, time series prediction, and gray theory prediction. However, these methods have some limitations in predicting the ship traffic volume, and the prediction results have large errors[2]. The neural network prediction model has a simple structure, the application method is relatively simple, and the neural network information synthesis ability is strong and the fault tolerance is high. The prediction results are relatively accurate. Wavelet neural network combines the advantages of wavelet analysis and neural network in dealing with nonlinear problems, and solves the blindness of BP neural network structure design and the problem of easy to fall into local optimum[3].Therefore, this paper establishes a genetic wavelet neural network prediction model and a BP neural network prediction model to predict the traffic volume of Jiujiang Port.

\section{Establishment of prediction model}

Prediction compared with the simple BP neural network Fan Qingbo used BP neural network and Markov model, constructed BP neural network-Markov prediction model, and optimized the model by particle swarm optimization. Finally, the monthly standard ship traffic flow of Wuhan Yangtze River Bridge was taken as a sample[4]. model, the conclusion that the BP neural network-Markov prediction model has higher accuracy is obtained. In addition, some scholars use wavelet neural network ${ }^{[5]}$ and other methods. This paper will use genetic algorithm and BP. The neural network and wavelet neural network are combined to construct the ship traffic flow prediction model, and it is applied to the ship traffic flow in Jiujiang Port. Then the results are compared, and the difference between the two methods in the prediction results and the method are discussed. Specific application.

\subsection{Establishment of genetic wavelet neural network prediction model}

\footnotetext{
${ }^{a}$ Corresponding author: 1036849174@qq.com
} 


\subsubsection{Wavelet neural network}

Wavelet neural network is a breakthrough by wavelet analysis in recent years, research on the basis of a kind of artificial neural network is put forward, which is based on the theory of wavelet analysis and wavelet transform is a kind of hierarchical structure made by new type of multiresolution and artificial neural network model, which USES nonlinear wavelet base instead of the usual nonlinear sigmoid function, the signal is described as table by the selection of wavelet base linear superposition, the corresponding input layer to hidden layer weights and threshold of hidden layer, respectively, by the expansion of the scale of the wavelet function is replaced by the translation factor and time factor. Wavelet function has good localization property and has penetrated into various fields. Wavelet transform is a time-frequency localization analysis method whose window size is fixed but whose shape, time window and frequency window can be changedm[6-7]. used because of its localized characteristics. Its basic principle is a layered, multiresolution new artificial neural network model composed of wavelet analysis and wavelet transform. Its model structure is shown in figure 1.

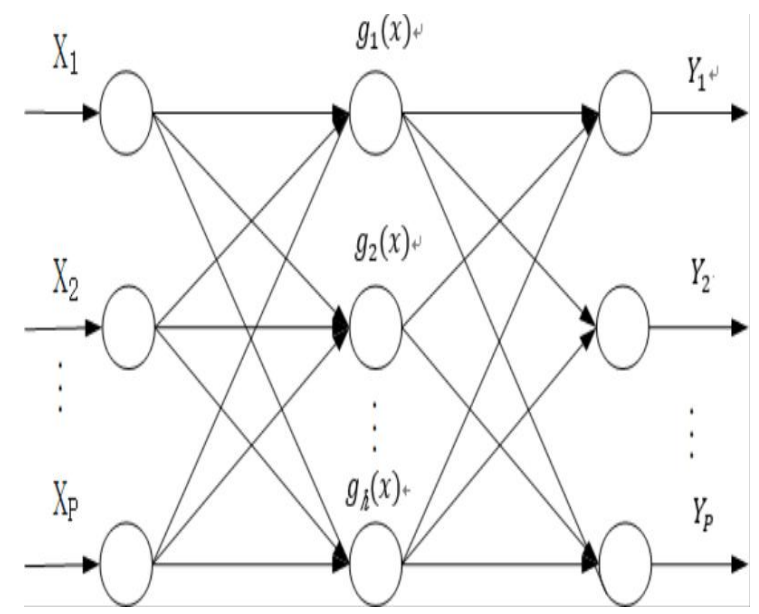

Figure 1. structure model of wavelet neural network

Wavelet neural network is a three-layer function, which is the input layer, hidden layer and output layer. Figure $\mathrm{X}$ shows the input sample of the input layer, $\mathrm{g}(\mathrm{x})$ is the wavelet function. It includes the scale scaling factor, the time shift factor, and $y$ is the output layer. The number of hidden layer units of the wavelet neural network can be adaptively determined as follows: First, take the wavelet network neural network hidden layer unit number $\mathrm{M}$ is 1 , after learning iteration several times, if it meets the error condition, stop iteration. If the maximum learning learning time is reached, the error condition is still not satisfied, then $\mathrm{M}+1$; repeat the above steps Until the error condition is met, this can overcome the blindness of the traditional neural network structure design.

\subsubsection{BP neural network}

BP neural network is also called error back propagation network, which has the advantages of strong learning ability, good nonlinear mapping ability and good fault tolerance. During the running of the algorithm, the information goes forward and the error propagates back to correct the network [8]. The algorithm core of the network is a step method (the steepest descent method). By optimizing the connection weight between layers, the sum of squared errors between the actual output value of the neural network and the ideal output is minimized.

\subsubsection{Genetic algorithm (ga)}

Genetic algorithm is a global optimization search algorithm that simulates the biological evolution process of genetic selection and natural elimination [9]. The operation of this algorithm has selection, crossover, mutation, and three operations. The genetic algorithm is simple to operate, and its parallel search ability is integrated into the nerve. In the network weight training of the network, it can search for the initial point with global ergodicity to ensure network training convergence and reduce training time. When genetic algorithm is applied to the optimization of network weights, its implicit parallel performance can overcome the difficulty that weights are easily trapped in local minima in the process of training, and it has the powerful function mapping and approximation ability of neural networks [10].

\subsection{Construction of prediction model}

\subsubsection{Construction of genetic wavelet neural network model}

Processing of input data

Based on common sense and basic statistical knowledge, the raw data is basically processed, the erroneous data is eliminated, and then each input data has different units and value dimensions, so each input data needs to be normalized.

Chromosome coding

The individual coding method is real number coding, and each individual is a real number string, which consists of an input layer and an intermediate layer link weight, an intermediate layer scale factor, a b, and an intermediate layer and an output layer connection weight. The individual contains all the weights and thresholds of the neural network. The total weights and thresholds in this paper are $5 \times 5+5+5+5 \times 1=40$, which is 40 -bit code. Wavelet function selection

For the selection of wavelet functions in wavelet networks, there is no unified theory or method to determine which type of wavelet function or scaling function is suitable as the excitation function of neurons in different networks, usually based on experience and actual situation. Of course, you can also learn from some of the experiences in wavelet analysis. In this paper, Morlet wavelet function is used as the excitation function of the network hidden layer by analyzing the literature and collecting data.

Fitness function 
According to the initial weight and threshold of the wavelet neural network, the training data is used to train the wavelet neural network and predict the output of the system. The sum of the absolute values of the errors between the predicted output and the expected output is taken as the individual fitness value $\mathrm{F}$. The calculation formula is :

$$
F=\sum_{i=1}^{n} a b s\left(y_{i}-o_{i}\right)=\pi r^{2}
$$

Where, $\mathrm{n}$ is the number of network output nodes; $y_{i}$ is the expected output of the i-th node of BP neural network, and $O_{i}$ is the predicted output of the $\mathrm{i}$-th node. The volume chart of ship traffic volume prediction algorithm is shown in figure 2 .

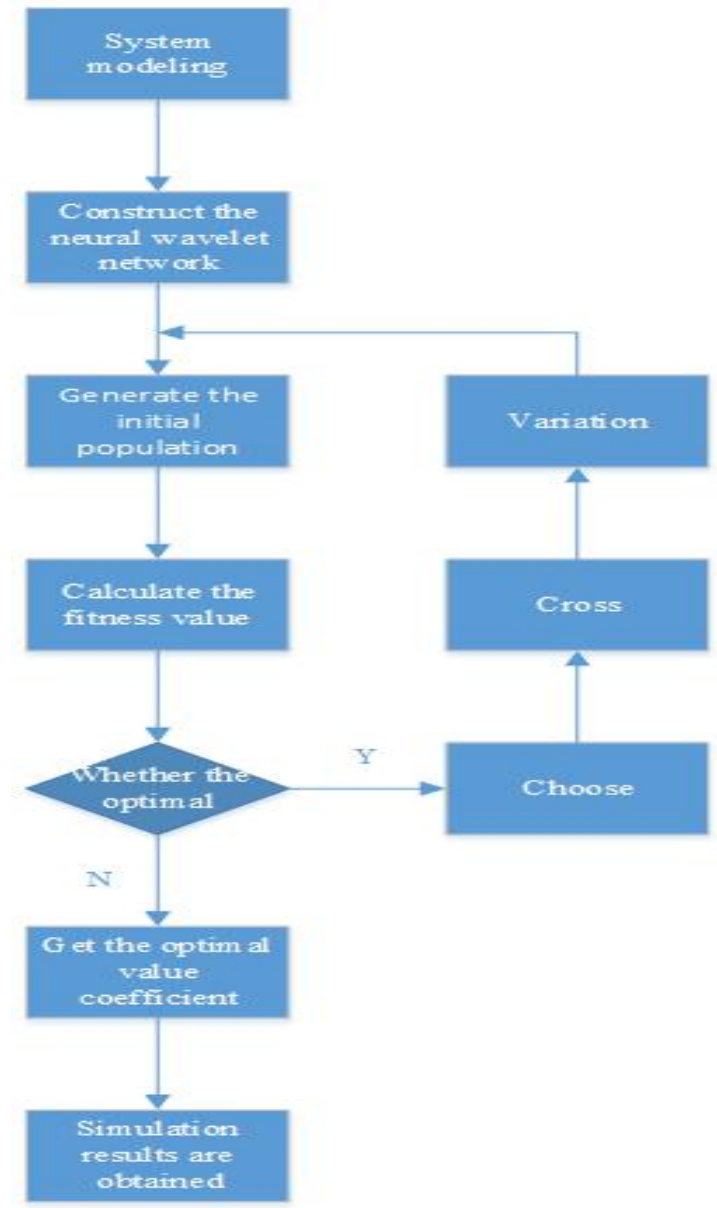

Figure 2 . genetic wavelet neural prediction model

\subsubsection{Construction of BP neural network model optimized by genetic algorithm}

Genetic algorithm optimization BP neural network is divided into three parts: BP neural network structure determination, genetic algorithm optimization and BP neural network prediction. The structure determining part of the BP neural network determines the BP neural network structure according to the number of input and output parameters of the fitting function, and then determines the length of the genetic algorithm individual. Genetic algorithm optimization uses genetic algorithm to optimize the weight and threshold of BP neural network. Each individual in the population contains all the weights and thresholds of a network. The individual calculates the fitness value of the individual through the fitness function. The genetic algorithm selects, crossover and mutation operations find the optimal fitness value corresponding to the individual. BP neural network prediction uses genetic algorithm to obtain the optimal individual's initial weight and threshold assignment to the network, and the network predicts the function output after training. The BP neural network model optimized by genetic algorithm is shown in Figure 3.

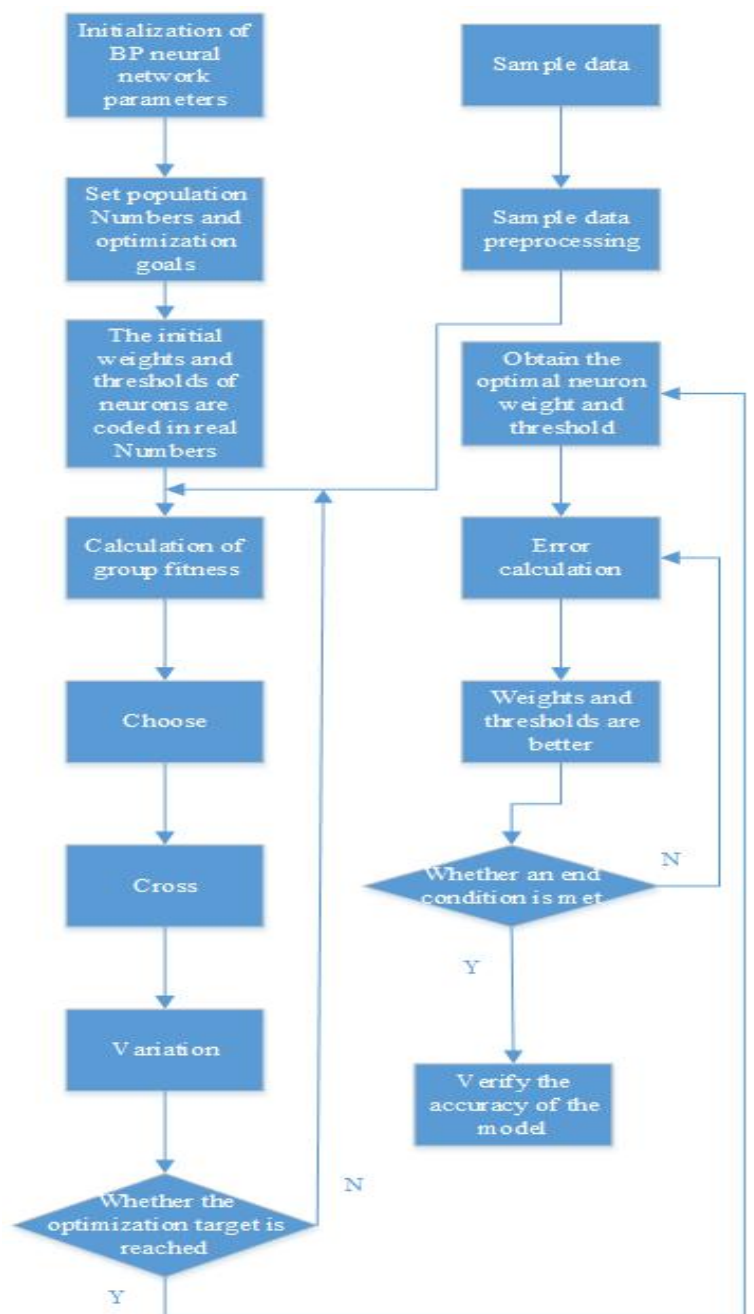

Figure 3. BP neural network model optimized by genetic algorithm

\subsection{Data sampling and processing}

Considering the many factors affecting the traffic volume of port ships, this paper uses the experimental data samples from January 2015 to January 2017 to predict the traffic volume of ships in Jiujiang Port in January 2018. In order to better characterize the concept of ship traffic volume, the port ship traffic volume has a more intuitive performance, and then accurately reflect the scale of the ship traffic volume in the waterway and the importance of the waterway, and convert the original data ship traffic volume into The weighted traffic volume of the ship, known as the standard ship, is also directly expressed as a standard ship. The conversion method is as shown in 
Table 1[11]. by the standard ship converted jiujiang port traffic volume are shown in table 2 .

Table 1 standard ship conversion coefficient table.

\begin{tabular}{l|c|c}
\hline $\begin{array}{c}\text { Gross tonnage of } \\
\text { ship }\end{array}$ & The captain & $\begin{array}{c}\text { Conversion } \\
\text { factor }\end{array}$ \\
\hline$<100$ & $<30$ & 0.25 \\
\hline $100 \sim 499$ & $30 \sim 50$ & 0.5 \\
\hline $500 \sim 2999$ & $50 \sim 90$ & 1 \\
\hline $3000 \sim 5999$ & $90 \sim 115$ & 1.18 \\
\hline $6000 \sim 9999$ & $115 \sim 135$ & 1.41 \\
\hline $10000 \sim 14999$ & $135 \sim 155$ & 1.7 \\
\hline $15000 \sim 19999$ & $155 \sim 170$ & 2.0 \\
\hline $20000 \sim 29999$ & $170 \sim 190$ & 2.25 \\
\hline $30000 \sim 39999$ & $195 \sim 215$ & 2.5 \\
\hline $40000 \sim 59999$ & $215 \sim 246$ & 3.0 \\
\hline$>60000$ & $>246$ & 4.0 \\
\hline
\end{tabular}

Table 2 ship traffic volume of jiujiang port from January 2015 to June 2017

\begin{tabular}{|l|l|l|l|l|l|}
\hline Time & \multicolumn{1}{|c|}{$\begin{array}{c}\text { The } \\
\text { sorting }\end{array}$} & $\begin{array}{c}\text { Vessel } \\
\text { traffic } \\
\text { volume } \\
\text { (standard } \\
\text { vessel) }\end{array}$ & Time & $\begin{array}{c}\text { The } \\
\text { sorting }\end{array}$ & $\begin{array}{c}\text { Vessel } \\
\text { traffic } \\
\text { volume } \\
\text { (standard } \\
\text { vessel) }\end{array}$ \\
\hline 2015.1 & 1 & 910 & 2016.4 & 16 & 1030 \\
\hline 2015.2 & 2 & 644 & 2016.5 & 17 & 919 \\
\hline 2015.3 & 3 & 918 & 2016.6 & 18 & 889 \\
\hline 2015.4 & 4 & 939 & 2016.7 & 19 & 940 \\
\hline 2015.5 & 5 & 922 & 2016.8 & 20 & 820 \\
\hline 2015.6 & 6 & 1037 & 2016.9 & 21 & 789 \\
\hline 2015.7 & 7 & 1028 & 2016.10 & 22 & 1050 \\
\hline 2015.8 & 8 & 1039 & 2016.11 & 23 & 1039 \\
\hline 2015.9 & 9 & 1015 & 2016.12 & 24 & 1080 \\
\hline 2015.10 & 10 & 1190 & 2017.1 & 25 & 1190 \\
\hline 2015.11 & 11 & 1020 & 2017.2 & 26 & 949 \\
\hline 2015.12 & 12 & 449 & 2017.3 & 27 & 920 \\
\hline 2016.1 & 13 & 1060 & 2017.4 & 28 & 1110 \\
\hline 2016.2 & 14 & 689 & 2017.5 & 29 & 1219 \\
\hline 15 & 1070 & 2017.6 & 30 & 1009 \\
\hline
\end{tabular}

\section{Simulation experiment and result research}

The simulation experiment was carried out in the MATLAB environment. The 24 sets of data in the 30 sets of port ship traffic volume data were network trained, and the remaining 6 groups were used as test data. The obtained neural network is trained by using the obtained training data to construct a neural network prediction model. The traffic volume data of the first six months of Jiujiang Port is selected to predict the traffic volume for the next month. The number of input nodes is 6 , and the output node is 1 . When the initial parameters of the neural network are optimized by GA, the maximum number of iterations is set to 100 , and the population size is 100 . The learning rates $\operatorname{lr} 1$ and $\operatorname{lr} 2$ are 0.01 and 0.001 , respectively. Then the results of the training were tested on the traffic volume of Jiujiang Port from January to June 2018 .

By using the model established by genetic neural network, the weights and correlation coefficients optimized by the genetic algorithm are assigned to the wavelet neural network. The absolute error percentage of the predicted and actual values of the test samples is used to evaluate the network prediction effect. Figure 4 is a graph of the absolute percentage of error for the network predictive output and expected output. It can be seen from Figure 4 that the prediction error values of the genetic wavelet neural network are distributed between 0.08 and 0.06 .

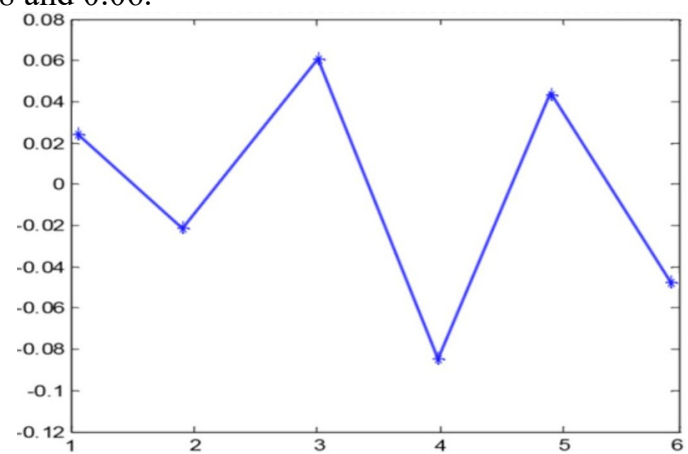

Figure 4. error percentage of prediction results of genetic wavelet neural network

Figure 5 below is the error result obtained by using Matlab operation of BP neural network optimized by genetic network. According to figure 5, the prediction error of genetic BP neural network are distributed between -0.15 and 0.3 .

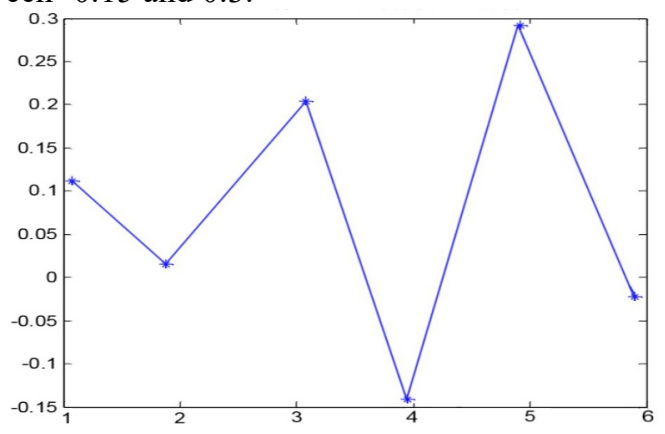


Figure 5. BP neural network result error percentage optimized by genetic algorithm.

It can be seen from Fig. 4 and Fig. 4 that the prediction error of genetic wavelet neural network is significantly smaller than that of BP neural network optimized by genetic algorithm. Using the trained genetic wavelet neural network and genetic algorithm optimized BP neural network to predict the ship traffic volume of Jiujiang Port from January to June 2018, the antinormalization of the prediction results, and the predicted ship traffic volume shown in Table 3 .

Table 3 forecasts ship traffic volume

\begin{tabular}{|l|c|l|l|l|l|}
\hline Time & $\begin{array}{l}\text { Actual } \\
\text { traffic } \\
\text { volume }\end{array}$ & $\begin{array}{l}\text { Wavelet } \\
\text { genetic } \\
\text { neural } \\
\text { predicti- } \\
\text { on }\end{array}$ & $\begin{array}{l}\text { Predic } \\
\text {-tion } \\
\text { error } \\
\text { /\% }\end{array}$ & $\begin{array}{l}\text { BP } \\
\text { genetic } \\
\text { neural } \\
\text { network } \\
\text { prediction }\end{array}$ & $\begin{array}{l}\text { Predic } \\
\text {-tion } \\
\text { error } \\
/ \%\end{array}$ \\
\hline $\begin{array}{l}2018- \\
01\end{array}$ & 842 & 824 & 2.1 & 934 & 11 \\
\hline $\begin{array}{l}2018- \\
02\end{array}$ & 481 & 490 & -2 & 476 & 1 \\
\hline $\begin{array}{l}2018- \\
03\end{array}$ & 833 & 783 & 6 & 666 & 20 \\
\hline $\begin{array}{l}2018- \\
04\end{array}$ & 782 & 845 & -8 & 899 & -15 \\
\hline $\begin{array}{l}2018- \\
05\end{array}$ & 783 & 814 & 4 & 548 & 30 \\
\hline $\begin{array}{l}2018- \\
06\end{array}$ & 799 & 832 & -4.2 & 823 & -3 \\
\hline
\end{tabular}

\section{Conclusion}

The analysis results of the above experiments show that the genetic wavelet neural network model is more accurate in predicting the traffic volume of the port than the genetic BP neural network prediction model, and the volatility in the prediction process is also small, which indicates the wavelet transform ratio. The traditional activation function in BP neural network is easier to obtain accurate prediction results. The wavelet neural network optimized by genetic algorithm tends to be globally optimal, and can improve the accuracy of prediction results.

In this paper, the characteristics of genetic algorithm are used to optimize the wavelet neural network, and its global fast optimization characteristics are combined with the characteristics of time-frequency locality of wavelet analysis to establish the genetic wavelet formed by genetic algorithm wavelet analysis and neural network. Neural network prediction models to predict port traffic volume. The experimental results show that the model has both the global fast optimization ability of genetic algorithm and the good time-frequency locality of wavelet transform, which can improve the prediction accuracy and propose an effective solution to solve the randomization problem of ship traffic volume

\section{References}

1. Zhang Renchu. Research on traffic volume prediction of ships inningbo port [D]. Dalian: dalian maritime university, 2008.

2. Li Hongxi, Fu Yuhui, Zhang Renchu. Prediction of port ship traffic volume [J]. Journal of dalian maritime university, 2009,35 (3) : 40-42.

3. HUANG Xiaohui, ZHANG Cuifang. Short-term traffic flow prediction based on cuckoo algorithm optimized wavelet neural network[J]. Computer Applications and Software, 2017, 34 (3): 238-242.

4. Fan Qingbo, Jiang Fucai, Ma Quanfang et al. BP neural network based on PSO-Markov ship traffic flow prediction model[J].Journal of Shanghai Maritime University, 2018,39(2):22-54.

5. [8] Meng Fanlin. Short-term prediction of ship traffic flow in VTS waters based on wavelet neural network[J]. China Maritime, 2017, (4): 45-47

6. Yu Xiaojiang, $\mathrm{Hu}$ Xuejun, $\mathrm{Hu}$ Yujin, et al. Intelligent adaptive control of vehicle spacing based on fuzzy neural network [J]. Journal of huazhong university of science and technology: natural science 2007, 35(9) : 22-24.

7. BARA A, DALE S, RUSU C, et al . DC Electrical Drive Control with Fuzzy System [ C ] Processing of the 13th International Conference on Engineering of Modern Electric System, ICEMES 2015, Oradea, Romania, 2015:207 - 201.

8. Wang Chaoxue, Kong Yueping, Dong Lili, et al. Intelligent Optimization Algorithm and Application [M]. Xi'an: North University Press, 2012.

9. Lu Feng, Xu Jianhua, Wang Zhanyong. Hefei City water demand prediction genetic wavelet nerve Network model research [J]. Surveying and Mapping Science, 2013, 38(5): 28-31.

10. Wang Caoxue, Kong Yue ping, etal. Intelligent optimizaton algorithm and its application [M]. Xi 'an: northwestern university press, 2012.

11. Wu haolin, Zhu Jun. Maritime traffic engineering [M]. Dalian: dalian maritime university press, 2004. 